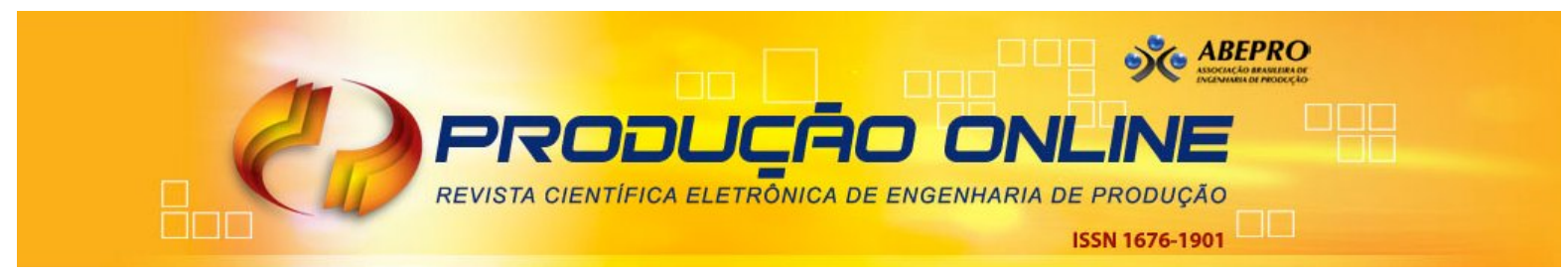

\title{
MANUTENÇÃO CENTRADA EM CONFIABILIDADE (MCC): ANÁLISE QUANTITATIVA DE UM FORNO ELÉTRICO A INDUÇÃO
}

\section{RELIABILITY CENTERED MAINTENANCE (RCM): QUANTITATIVE ANALYSIS OF AN INDUCTION ELECTRIC FURNACE}

\author{
Diego Santos Cerveira* E-mail: cerpa2001@hotmail.com \\ Miguel Afonso Sellitto* E-mail: sellitto@unisinos.br \\ *Universidade do Vale do Rio dos Sinos (UNISINOS), São Leopoldo, RS
}

\begin{abstract}
Resumo: O objetivo deste artigo é definir uma estratégia de manutenção para um forno elétrico a indução, instalado em uma fundição de aços especiais. O método de pesquisa foi a modelagem quantitativa. O método proposto é baseado na metodologia da Manutenção Centrada na Confiabilidade (MCC), aplicada a equipamento industrial. Análises quantitativas de confiabilidade, mantenabilidade e disponibilidade foram usadas como ferramentas de apoio à definição da estratégia de manutenção do equipamento. Para a pesquisa, foram coletados dados históricos de tempo até o reparo (TTR) e tempo entre falhas (TBF) do equipamento em estudo. Com o auxílio do software ProConf 2000, foram identificadas e modeladas as distribuições mais adequadas para esses tempos: lognormal (TTR) e Weibull (TBF). Com os valores obtidos foi possível calcular a disponibilidade do equipamento, $A v=$ $98,18 \%$, e o fator de forma $\gamma=1$ da distribuição de Weibull. Foi possível posicionar o equipamento na curva da banheira, na fase de maturidade e definir a melhor estratégia de manutenção para este caso, a preditiva. Por fim, discutiu-se a estratégia adotada atualmente pela empresa e apresentaram-se sugestões de desenvolvimento para esta estratégia.
\end{abstract}

Palavras-chave: Confiabilidade. Mantenabilidade. Disponibilidade. Estratégia de Manutenção.MCC.

Abstract: The purpose of this article is to define a maintenance strategy for an electric induction furnace, installed on a special steels Foundry. The research method was the quantitative modeling. The proposed method is based on Reliability-Centered Maintenance (RCM), applied to industrial equipment. Quantitative analysis of reliability, availability and maintainability were used as support the definition of the maintenance strategy of the equipment. For research, historical data were collected from time-torepair (TTR) and time between failures (TBF) of the equipment under consideration. Supported by ProConf 2000 software, most appropriate distributions have been identified and modeled to TTR (lognormal) and TBF (Weibull). With the results, availability of equipment Av $=98,18 \%$ and form factor $\gamma=1$ of the Weibull distribution were calculated. It was possible to find a position for the equipment on the bathtub curve, in the maturity phase and define the best maintenance strategy for this case, the predictive maintenance. Finally, the current strategy was discussed and development suggestions were presented to this strategy.

Keywords: Reliability. Maintainability. Availability. Maintenance Strategy. RCM.

\section{INTRODUÇÃO}

Historicamente, a manutenção tem sido vista como fator gerador de custos para as empresas. Porém, no cenário atual, de economia globalizada, a competitividade 
crescente em manufatura tem exigido melhorias e redução de custos em processos produtivos, o que tem aumentado o uso de equipamentos e processos, aumentando a importância da manutenção (SIQUEIRA, 2009). A estratégia de manutenção passou, então, a ser fator de construção de vantagem competitiva em manufatura (MENDES, 2011), pois esta depende da produtividade, que é influenciada pela disponibilidade do sistema de produção (RAPOSO, 2011; RAMOS FILHO et al., 2010). Tais argumentos reforçam a importância estratégica da função manutenção na atual gestão da manufatura.

A indisponibilidade de equipamentos críticos pode interferir no desempenho fabril (FAGUNDES et al., 2011), o que pode ser medido e controlado por indicadores adequados (PACHECO et al., 2012). Slack et al. (2010) afirmam que nem todas as falhas são igualmente danosas: a importância da falha é determinada por seu efeito no desempenho global do sistema de produção. Se algumas falhas podem não ter efeito, outras podem gerar desperdício de matéria-prima em processo, afetar a qualidade dos produtos ou atrasar prazos de entrega, causando aumento de custos, insatisfação dos clientes e danos à imagem da empresa.

Essas características são compatíveis com o que se observa em uma fundição de aços especiais de grande porte, tal como a investigada neste artigo. Nesse tipo de empresa, as corridas de produção são longas, os lotes são grandes, o produto é sempre o mesmo, e há pouca variação na matéria prima. Neste cenário, falhas em alguns equipamentos podem causar muito mais danos do que em outros, dada a característica de processo em linha (SELLITTO, 2002).

Em casos deste tipo, é importante que os equipamentos críticos tenham ao mesmo tempo altas confiabilidade e mantenabilidade (ou manutenibilidade), e por consequência alta disponibilidade. Confiabilidade é a probabilidade que um sistema de produção opere isento de falhas por um dado tempo. Mantenabilidade é a probabilidade que, dado que ocorreu uma falha, o reparo seja feito até um dado tempo (FOGLIATTO e RIBEIRO, 2009). Disponibilidade é a relação entre o tempo que o recurso está disponível e o tempo total previsto para operação. Seu cálculo deriva da confiabilidade e da mantenabilidade (CORREA e CORREA, 2010). A disponibilidade é um dos principais indicadores utilizados em manutenção (FOGLIATTO e RIBEIRO, 2009).

Para gerenciar a confiabilidade e a disponibilidade de equipamentos, a gestão da manutenção pode usar métodos qualitativos ou quantitativos. Métodos qualitativos 
incorporam fatores subjetivos nas análises. Nos métodos quantitativos, as previsões são baseadas em dados históricos dos quais se procura extrair padrões de comportamento (CORRÊA e CORRÊA, 2010). Para Kuyven (2010), a principal função dos métodos quantitativos é gerar informações que levem à compreensão do objeto de estudo e ao estudo das opções diante das tomadas de decisão. Este artigo limitouse ao uso de métodos quantitativos.

Sellitto (2007) e Kardec e Nascif (2001) apontam a Manutenção Centrada na Confiabilidade (MCC) como um dos métodos de suporte a decisões capazes de contribuir para o aumento da confiabilidade na produção. De modo geral, a literatura sobre MCC aborda mais aspectos qualitativos do que quantitativos, porém são as técnicas quantitativas que podem contribuir mais efetivamente para o aumento da confiabilidade dos sistemas de produção (MENDES, 2011). Portanto, pode ser de interesse o uso de técnicas quantitativas no apoio a decisões centrais em manutenção, tais como a formulação de uma estratégia.

O objetivo deste artigo é definir uma estratégia de manutenção para um forno elétrico a indução, instalado em uma fundição de aços especiais. Os objetivos secundários são: calcular as funções confiabilidade e mantenabilidade; pelo fator de forma do modelo de confiabilidade, posicionar o equipamento no ciclo de vida (curva da banheira); pelos valores esperados das duas funções, calcular a disponibilidade do equipamento; e comparar a estratégia atua com a estratégia indicada pelos resultados da pesquisa. $O$ método de pesquisa foi a modelagem quantitativa.

Casos semelhantes foram encontrados na literatura. Desses, alguns contribuíram para este artigo e podem ser citados. Fagundes et al. (2011) aplicaram distribuições de probabilidade para analisar a relação entre a taxa de falha de um torno CNC e a estratégia de manutenção adotada. Silva e Anzanello (2012) propuseram um intervalo ideal de manutenções preventivas em um processo de calandragem visando reduzir refugos. Reis e Andrade (2009), com o auxílio de softwares de análise de confiabilidade, modelaram o comportamento das falhas de um molde de injeção de polímeros e estabeleceram a estratégia e os intervalos de manutenção a adotar. Bernardes (2006) propôs um método de auxílio à decisão e avaliação de riscos no gerenciamento de linhas de transmissão de energia elétrica baseado em modelos probabilísticos dos tempos entre falhas e até o reparo. Dantas et al. (2010) utilizaram o modelo de regressão de Weibull para analisar o risco de falha de poços petrolíferos. Foi verificada a relação entre o tempo até a primeira falha e algumas características 
dos poços, tais como: idade, profundidade e volume de produção. Sellitto (2005) propôs um método objetivo para formular estratégias de manutenção de equipamentos industriais a partir das análises de confiabilidade e mantenabilidade. Girelli et al. (2006) utilizaram tempos entre falhas e até o reparo para, segundo o modelo de Weibull, desenvolver um simulador computacional para gestão da manutenção, que resulta na melhor combinação de peças de reposição. Wuttke e Sellitto (2008) e Mengue e Sellitto (2013) aplicaram funções de confiabilidade em equipamentos petroquímicos e petrolíferos. Santos et al. (2007) construíram um modelo, baseado no modelo de Poisson não-homogêneo, para estimar o intervalo ótimo entre manutenções preventivas para sistemas sujeitos a reparos mínimos. Sellitto et al. (2002) aplicaram um método baseado em confiabilidade para gestão da manutenção de equipamentos de transporte coletivo.

O restante do artigo está estruturado em: referencial teórico; descrição da pesquisa; discussão dos resultados; e considerações finais.

\section{CONFIABILIDADE E MANUTENÇÃO INDUSTRIAL}

A Manutenção Centrada na Confiabilidade (MCC) originou-se na indústria aeronáutica americana, porém seus conceitos e técnicas são aplicáveis a outros setores da indústria (SIQUEIRA, 2009). A MCC pode ser definida como um programa que reúne várias técnicas de engenharia para assegurar que os equipamentos de uma planta fabril cumprirão suas funções especificadas. O modelo binário é o mais simples usado para representar a condição de um item ou sistema: em funcionamento ou em falha (FOGLIATTO e RIBEIRO, 2009).

Para Lafraia (2001), o objetivo da manutenção na ótica da MCC é assegurar que um sistema ou item continue a cumprir suas funções desejadas, o que a diferencia do enfoque tradicional. Na MCC, as falhas são tratadas conforme seu potencial de geração de risco para o resultado da operação produtiva. No enfoque tradicional, toda falha é indesejável e deve ser prevenida. Lafraia (2001) caracteriza este enfoque como inviável por duas razões: (i) tecnicamente, é impossível evitar todas as falhas; e (ii) ainda que fosse possível, os recursos disponíveis não seriam suficientes.

Fogliatto e Ribeiro (2009) identificam como requisito prévio indispensável para a MCC a disponibilidade de informações. Para tanto, é essencial estabelecer um banco de dados que registre e classifique as falhas observadas no sistema, o que 
permite estudos formais de confiabilidade que servirão de base para 0 dimensionamento das atividades de manutenção.

\subsection{Funções de confiabilidade}

A confiabilidade de um equipamento ou sistema é definida na fase de projeto, mas pode ser gerenciada pela manutenção (CUNHA et al., 2012). As funções mais utilizadas para análise da confiabilidade são: função densidade de falhas $f(t)$, função acumulada de falhas $F(t)$, função confiabilidade $R(t)$ e função de risco $h(t)$. As expressões que seguem são apresentadas em Fogliatto e Ribeiro (2009).

A função densidade de falhas $f(t)$ representa a variação da probabilidade de falhas por unidade de tempo. É representada graficamente por uma função de distribuição de probabilidade e matematicamente representada pela equação (1).

$$
f(t)=\frac{d F(t)}{d t}
$$

$F(t)$, a função acumulada de falhas, calcula a probabilidade de falha entre um período $t_{1}$ e $t_{2}$. É representada graficamente por uma função de distribuição de densidade acumulada e matematicamente expressa pela equação (2).

$$
F\left(t_{2}\right)-F\left(t_{1}\right)=\int_{t_{1}}^{t_{2}} f(t) d t
$$

A função confiabilidade $R(t)$ é dada pela equação (3) e calcula a probabilidade de que um item não falhe em um dado período.

$$
R(t)=\int_{t}^{\infty} f(t) d t=1-\int_{-\infty}^{t} f(t) d t=1-F(t)
$$

A função de risco $h(t)$ é dada pela equação (4) e descreve a probabilidade condicional de falha no intervalo de $[t ; t+\Delta t]$, dado que não houve falha em $t$.

$$
h(t)=\frac{f(t)}{C(t)}=\frac{f(t)}{1-F(t)}
$$


Para estudos de confiabilidade em manutenção, é necessário determinar uma distribuição de probabilidade que se ajuste aos dados de sobrevivência do sistema até o tempo desejado. As principais distribuições de interesse para a manutenção são: lognormal, exponencial, gamma e Weibull (FOGLIATTO e RIBEIRO, 2009). Os autores também citam a distribuição normal como relevante na estatística inferencial, mas com pouca aplicabilidade em análises de confiabilidade.

A distribuição lognormal é mais usada em peças de desgaste, tais como componentes metálicos sujeitos à falha por fadiga do metal, trincas ou corrosão. As medidas de confiabilidade de interesse para a distribuição lognormal são dadas pelas equações de (5) a (8), para $t \geq 0$ (LAFRAIA, 2001; FOGLIATTO e RIBEIRO, 2009; GROSH, 1989):

$$
\begin{array}{r}
f(t)=\frac{1}{\sigma \cdot t \sqrt{2 \pi}} e^{\left[-\frac{1}{2}\left(\frac{\ln t-\mu}{\sigma}\right)^{2}\right]} \\
R(t)=\Phi\left(\frac{\mu-\ln t}{\sigma}\right) \\
h(t)=\frac{\phi[(\mu-\ln t) / \sigma] / \sigma t}{\Phi[(\mu-\ln t) / \sigma]} \\
\text { MTTF ou MTBF=e } e^{\left(\mu+\sigma^{2}\right) / 2}
\end{array}
$$

\section{Em que:}

$\Phi(x)=$ valor da função de distribuição da distribuição normal avaliada em $x$;

$\phi(x)=$ valor da função de densidade da distribuição normal avaliada em $x$;

$\mu=$ média do logaritmo dos dados;

$\sigma=$ desvio padrão do logaritmo dos dados;

$t=$ tempo até a falha.

A distribuição exponencial é uma distribuição contínua com taxa de falhas constante, sendo utilizada para descrever falhas em sistemas complexos não redundantes ou sistemas complexos com taxas de falhas independentes. Também pode ser aplicada em sistemas com dados de falhas mostrando causas heterogêneas 
ou sistemas com muitos componentes, com substituições antes de falhas devido à manutenção preventiva. Suas representações de confiabilidade são expressas pelas equações de (9) a (12), para $t \geq 0$ (FOGLIATTO e RIBEIRO, 2009; LEWIS, 1996; DODSON e NOLAN, 2002):

$$
\begin{array}{r}
f(t)=\lambda e^{-\lambda t} \\
R(t)=e^{-\lambda t} \\
h(t)=\lambda \\
\text { MTTF ou MTBF }=1 / \lambda
\end{array}
$$

Em que:

$\lambda=$ taxa de falha;

$t=$ tempo até $a$ falha.

A distribuição gamma é uma generalização da distribuição exponencial. É utilizada para descrever sistemas que operam com componentes em paralelo, em que todos os componentes devem falhar para que o sistema falhe. As funções de confiabilidade são expressas pelas equações de (13) a (16, para $t \geq 0, \gamma>0$ e $\lambda>0)$ (LAFRAIA, 2001; FOGLIATTO e RIBEIRO, 2009; GROSH, 1989):

$$
\begin{array}{r}
f(t)=\frac{\lambda}{\Gamma(\gamma)}(\lambda t)^{y-1} e^{-\lambda t} \\
R(t)=1-\frac{1}{\Gamma(\gamma)} \int_{0}^{\lambda t} x^{y-1} e^{-x} d x \\
h(t)=\frac{f(t)}{R(t)} \\
\text { MTTF ou MTBF }=\gamma / \lambda
\end{array}
$$


Em que:

$\Gamma=$ função gamma;

$\gamma=$ parâmetro de forma;

$\lambda=$ parâmetro de escala;

$t=$ tempo até a falha.

A distribuição de Weibull é uma das mais importantes distribuições em modelos de confiabilidade devido à flexibilidade e capacidade de representação de amostras de tempos até a falha com comportamentos distintos, além de poder ser usada em pequenas amostras (LAFRAIA, 2001; FOGLIATTO e RIBEIRO, 2009; LEWIS, 1996; DODSON, 1994). O modelo de Weibull é indicado para explicar o comportamento de sistemas cuja falha nasce da competição entre diversos modos de falha. Quando os modos de falha atuam em série, o primeiro componente a falhar causa a falha do sistema. As medidas de confiabilidade, para $t \geq 0, \gamma>0$ e $\theta>0$, são expressas pelas equações de (17) a (20) (LAFRAIA, 2001; FOGLIATTO e RIBEIRO, 2009; LEWIS, 1996; DODSON, 1994).

$$
\begin{array}{r}
f(t)=\frac{\gamma}{\theta \vartheta} t^{y-1} e^{-t^{\gamma} / \theta} \\
R(t)=e^{-\left(\frac{t}{\theta}\right)^{\gamma}} \\
h(t)=\frac{\gamma}{\theta}\left(\frac{t}{\theta}\right)^{\gamma-1} \\
\text { MTTF ou MTBF }=\theta \Gamma(1+1 / \gamma)
\end{array}
$$

Em que:

$\theta=$ parâmetro de escala;

$\gamma=$ parâmetro de forma;

$\Gamma=$ função gamma;

$t=$ tempo até a falha. 
A distribuição de Weibull modela uma ampla variedade de situações em que unidades apresentam funções de risco distintas (LAFRAIA, 2001). A Tabela 1 descreve o comportamento da função de risco $h(t)$ segundo o parâmetro de forma $\gamma$.

Tabela 1 - Comportamento da função taxa de falhas

\begin{tabular}{cl}
\hline$\gamma$ & Comportamento da função $\boldsymbol{h}(\boldsymbol{t})$ \\
\hline$<1$ & Taxa de falha decrescente \\
$=1$ & Taxa de falha constante - distribuição exponencial \\
$>1$ & Taxa de falha crescente \\
$=2$ & Taxa de falha linearmente crescente - distribuição de Rayleigh \\
$=3,2$ & Distribuição de frequência similar ao da distribuição normal \\
\hline
\end{tabular}

Fonte: Adaptado de Lafraia (2001)

Podem-se destacar dois casos especiais da distribuição de Weibull: (i) quando o parâmetro de forma $\gamma=2$, a distribuição de Weibull se transforma na distribuição de Rayleigh; e (ii) quando o parâmetro de forma $\gamma=1$, a distribuição de Weibull se reduz a uma exponencial, como no caso apresentado neste artigo. Mais considerações sobre a distribuição de Weibull e suas variantes podem ser encontradas em Dodson (1994) e para as demais distribuições em Grosh (1989), Dodson e Nolan (2002) e Lewis (1996). Mais considerações sobre probabilidade e modelos estatísticos aplicados à engenharia podem ser encontrados em Nelson (1982), Lawless (2003) e Montgomery et al. (2011).

\subsection{Taxa de falhas e estratégias de manutenção}

O comportamento da taxa de falha de um equipamento ao longo do tempo pode ser analisado pela curva da banheira (bathtub curve), que representa genericamente a função de risco $h(t)$ ao longo do ciclo de vida. A curva da banheira, representada na Figura 1, apresenta três períodos característicos de vida de componentes e equipamentos: mortalidade infantil, fase de maturidade, e mortalidade senil (LAFRAIA, 2001; SELLITTO, 2005). 
Figura 1 - Curva da banheira e o ciclo de vida dos equipamentos

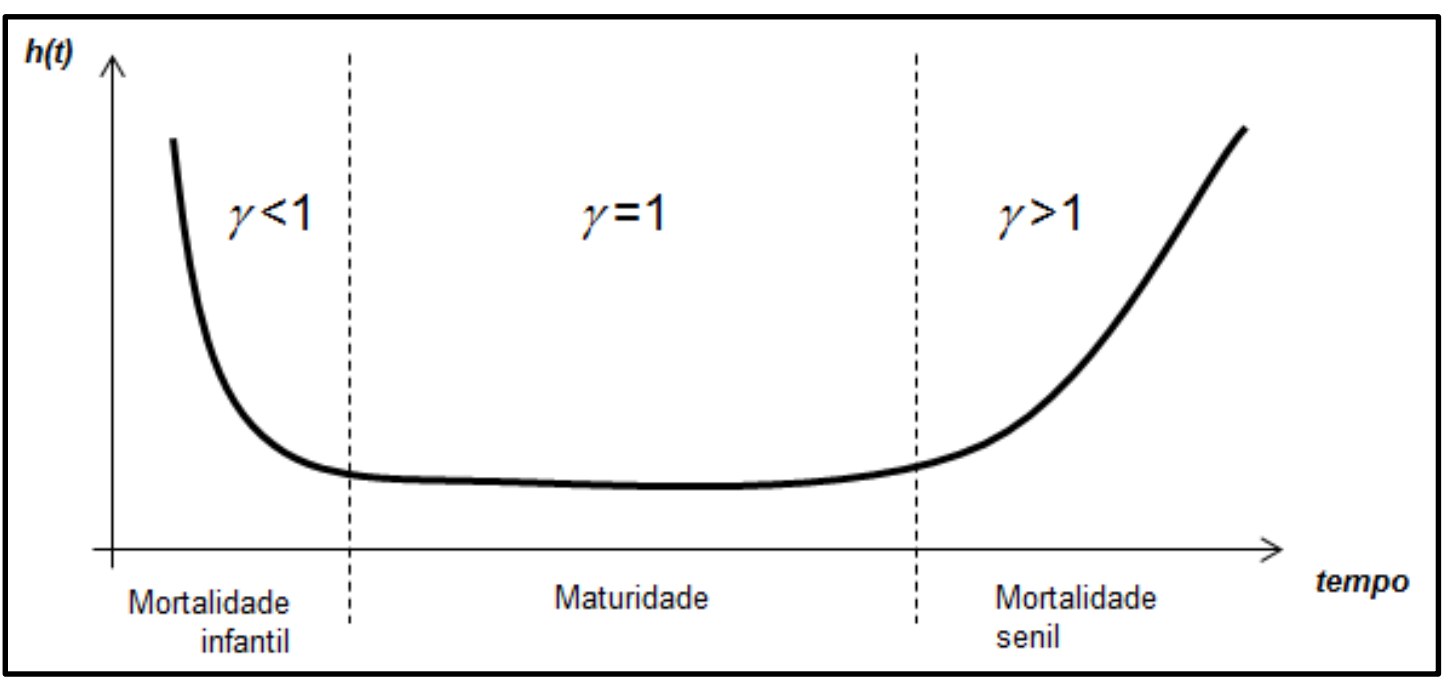

Fonte: Adaptado de Lafraia (2001) e Sellitto (2005)

Sellitto (2005) relaciona cada fase da curva a um comportamento da função de risco $h(t)$, pelo fator de forma $\gamma$ da distribuição de Weibull que descreve a função confiabilidade do equipamento. O autor também associa a cada fase da curva uma estratégia de manutenção mais adequada.

No período inicial, chamado de mortalidade infantil, a taxa de falhas é alta, porém decrescente. As falhas são prematuras, normalmente originadas por deficiências no processo de fabricação, instalação incorreta, ou materiais fora de especificação (LAFRAIA, 2001). A estratégia para esta fase é a manutenção corretiva, que identifica e corrige deficiências de projeto ou de instalação do equipamento (SELLITTO, 2005). A estratégia de manutenção corretiva também se justifica quando eventuais falhas não são críticas para a produção (MONCHY, 1989).

No período seguinte, denominado fase de maturidade por Sellitto (2005) e de vida útil por Kardec e Nascif (2001), a taxa de falhas é sensivelmente menor e oscila ao redor de uma média constante. As falhas são casuais e decorrentes de fatores menos controláveis, tais como: mau uso do equipamento, ultrapassagem de resistência ou fenômenos naturais imprevisíveis. A estratégia para esta fase é a manutenção preditiva. Tal tipo de manutenção é realizado conforme a necessidade, baseado no resultado de inspeções contínuas ou periódicas. Quando o grau de degradação atinge o limite estabelecido, uma intervenção de manutenção é executada antes da falha (SELLITTO, 2005). Esta estratégia também é indicada quando os 
custos de manutenção ou de parada da produção forem elevados (CORRÊA e CORRÊA, 2010).

O último período da curva é chamado de desgaste por Lafraia (2001) e de mortalidade senil por Sellitto (2005). Segundo os autores, é o fim da vida útil do equipamento. No período, a taxa de falhas é crescente. Essas falhas são causadas por envelhecimento, degradação mecânica, elétrica ou química, fadiga, corrosão, ou vida de projeto muito curta. A estratégia para esta fase é a manutenção preventiva: a troca antecipa a inevitável quebra (SELLITTO, 2005). A manutenção preventiva também é conveniente quando as falhas prejudicarem os compromissos de entrega produção ou quando os custos da quebra forem altos (KARDEC e NASCIF, 2001).

\subsection{Mantenabilidade e disponibilidade}

A função mantenabilidade está relacionada ao projeto de um equipamento. È na fase de projeto que são determinados os procedimentos e os tempos necessários para a manutenção. Ireson et al. (1996) relacionam as definições de confiabilidade e mantenabilidade. Enquanto a primeira tenta reduzir a frequência ou a severidade das falhas em sistemas, a segunda concentra-se em reduzir o tempo de duração das falhas e de recolocação do sistema em funcionamento.

A Associação Brasileira de Normas Técnicas (ABNT), pela NBR 5462 (1994), define mantenabilidade como sendo a facilidade de um item em ser mantido ou recolocado no estado necessário para executar suas funções requeridas, sob condições determinadas e mediante procedimentos e meios prescritos e conhecidos. A mantenabilidade é definida como a probabilidade do equipamento ser recolocado em condições plenas de operação dentro de um dado período de tempo quando as ações de manutenção são executadas de acordo com procedimentos preestabelecidos (LAFRAIA, 2001).

Assim como para as funções de confiabilidade, é necessário determinar uma distribuição de probabilidade para a mantenabilidade. As distribuições mais utilizadas são a normal e a lognormal. Para Mengue e Sellitto (2013), a normal se aplica quando o reparo é composto pela soma de tarefas independentes e sequenciais, como ocorre na desmontagem e remontagem de máquinas em reformas ou manutenções preventivas. Já a distribuição lognormal se ajusta a casos em que as atividades de 
manutenção envolvem componentes intelectuais e cognitivos, como no caso estudado neste artigo.

A mantenabilidade pode descrever quantitativamente as atividades de reparo pelo tempo médio que a equipe de manutenção levará para recolocar o equipamento em funcionamento. Este tempo $T$ não é constante, pois varia devido a fatores que vão da capacidade em diagnosticar a falha até a disponibilidade de equipamentos e a habilidade para realizar o reparo. O tempo $T$ é uma variável randômica. A função densidade de probabilidade de reparo $m(t)$ é expressa pela equação (21), sendo $m(t)$ a probabilidade que o reparo seja realizado no intervalo [t; $(t+\Delta \mathrm{t})]$. A função distribuição de reparo $M(t)$ é apresentada na equação (22) e o tempo médio até o reparo (MTTR) em (23) (LEWIS, 1996).

$$
\begin{array}{r}
m(t) . \Delta t=P\{t \leq T \leq t+\Delta t\} \\
M(t)=\int_{0}^{t} m(t) d t \\
M T T R=\int_{0}^{\infty} t \cdot m(t) d t
\end{array}
$$

A disponibilidade de equipamentos é um dos principais indicadores de confiabilidade utilizados na manutenção. Fogliatto e Ribeiro (2009) e Lafraia (2001) definem disponibilidade $A v(t)$ como a probabilidade de que um equipamento ou sistema esteja em condição operacional quando necessitado. Para Corrêa e Corrêa (2010), a disponibilidade $A v(t)$ é uma relação entre o tempo disponível de um equipamento e o tempo total previsto para a sua operação. O tempo disponível é o tempo entre duas falhas sucessivas ou Mean Time Between Failures (MTBF) e o tempo total é tempo disponível mais o tempo até o reparo ou Mean Time To Repair (MTTR). Considerando que se tenham o MTBF e o MTTR, a disponibilidade pode ser expressa pela equação (24).

$$
A v(t)=\frac{\text { Tempo }_{\text {disponível }}}{\text { Tempo }_{\text {total }}}=\frac{M T B F}{M T B F+M T T R}
$$




\section{PROCEDIMENTOS METODOLÓGICOS}

O método de pesquisa foi a modelagem quantitativa. Modelagem ou modelamento compreende o uso de técnicas matemáticas para descrever o comportamento de um objeto de estudo (NAKANO, 2010; MIGUEL, 2007). Se for possível descrever o comportamento de um objeto por um modelo matemático, por tratamento matemático adequado, também é possível extrair conclusões a seu respeito. A modelagem é citada como método válido em Engenharia de Produção por Nakano (2010) e foi reconhecida anteriormente como método de pesquisa em gestão de operações por Filippini (1997). Seus detalhes e características, em conjunto com simulação, incluindo aplicações em pesquisa operacional, são abordados por Morabito e Pureza (2010). A pesquisa se limitou à análise quantitativa por modelagem de duas variáveis aleatórias: tempo entre falhas e tempo até o reparo. Martins (2010) aponta modelagem e simulação com um dos quatro métodos aplicáveis em pesquisas quantitativas em Engenharia de Produção.

A metodologia do trabalho foi baseada em Miguel (2010):

- revisão bibliográfica dos conceitos de confiabilidade e mantenabilidade aplicados à manutenção de equipamentos industriais, de modo a definir uma estrutura teórico-conceitual;

- planejamento e delimitação do sistema a ser estudado: um forno a indução decisivo para o resultado de uma fundição de aços especiais (pelo teste-piloto, percebeu-se que os dados disponíveis eram confiáveis e se ajustavam a distribuições conhecidas, o que tornou o estudo viável);

- obtenção dos dados dos tempos entre falhas e tempos até o reparo do forno;

- análise dos dados por modelagem pelas distribuições gamma, normal, lognormal, exponencial e Weibull, pelo software ProConf 2000;

- definição da distribuição que melhor se ajusta aos dados com base nos testes de máxima verossimilhança e na premissa da pesquisa;

- identificação do ponto no ciclo de vida em que o equipamento se encontra;

- cálculo das funções confiabilidade e mantenabilidade e disponibilidade; e

- discussão dos resultados, análise da estratégia atual e proposta de estratégia de manutenção mais adequada ao equipamento em estudo. 


\section{LEVANTAMENTO E ANÁLISE DE DADOS}

A condição básica para a realização da análise de falhas é que exista um banco de dados com informações de manutenção suficientes e factíveis.

A partir do sistema de informação da empresa, foram coletados e analisados dados históricos referentes à manutenção do forno entre janeiro e dezembro de 2012. Foram consideradas apenas as paradas não programadas, ou seja, quebras de equipamentos. Foram coletados os dados de tempo até o reparo ou Time to Repair (TTR) e tempo entre falhas ou Time Between Failures (TBF). Como premissa de pesquisa assume-se (SELLITTO, 2005): (i) se os TTR se ajustarem a uma distribuição lognormal, as demais serão descartadas; e (ii) se os TBF se ajustarem a uma distribuição Weibull, as demais serão descartadas; se se ajustarem também a uma exponencial, esta última será adotada.

O forno em estudo foi instalado em 2009 e é revisado anualmente, durante o período de férias coletivas da empresa. As informações pertinentes às atividades de manutenção são registradas no sistema de informação da empresa. Para este artigo interessam os TTR e TBF, apresentados respectivamente nas Tabelas 2 e 3.

Tabela 2 - Histórico de dados (TTR)

\begin{tabular}{cccc}
\hline \multicolumn{4}{c}{ Tempo até o reparo (minutos) } \\
\hline 50 & 60 & 75 & 20 \\
90 & 150 & 120 & 495 \\
600 & 150 & 60 & 120 \\
50 & 80 & 180 & 90 \\
180 & 310 & 120 & 135 \\
\hline Fonte: Sistema de informação da empresa \\
\multicolumn{4}{c}{} \\
Tabela 3 Histórico de dados (TBF) \\
\hline \multicolumn{4}{c}{ Tempo entre falhas (minutos) } \\
\hline 4860 & 540 & 7020 \\
3240 & 1620 & 12960 & 17280 \\
1080 & 23220 & 2700 & 1620 \\
3780 & 12420 & 22680 & 2700 \\
10800 & 2160 & 2700 & 16200 \\
\hline
\end{tabular}

Fonte: Sistema de informação da empresa 
O forno elétrico a indução é um tipo forno de fusão que utiliza correntes elétricas para derreter o metal. Essas correntes são alternadas através de bobinas elétricas que contornam o cadinho que contém o metal, aquecendo-o até a temperatura necessária para sua fusão. Essa temperatura é pré-determinada de acordo com matéria-prima em uso. O forno é equipado com um transformador e um conversor de frequência, que regulam a tensão e a frequência de operação, necessários para o seu funcionamento. Durante o funcionamento do forno as bobinas elétricas precisam ser resfriadas para que não superaqueçam e se danifiquem. Para isso, o forno conta com um sistema próprio de arrefecimento do sistema elétrico. Esses fornos possuem ainda um sistema hidráulico de basculamento e um sistema de exaustão de gases produzidos na fusão dos metais.

A Tabela 4 apresenta os principais dados técnicos do forno em estudo.

Tabela 4 - Forno elétrico a indução

\begin{tabular}{lc}
\hline \multicolumn{2}{c}{ Características técnicas } \\
\hline Modelo & MF 3 / 3 / 1500 Bl-WORK \\
\hline Capacidade de carga & $6000 \mathrm{~kg}$ \\
$\mathrm{~N}^{0}$ de cubas & $2(3000 \mathrm{~kg} / \mathrm{cada})$ \\
Potência Nominal & $1500 \mathrm{~kW}$ \\
Frequência de operação do forno & $1000 \mathrm{~Hz}$ \\
Temperatura de operação & $1450-1650$ C \\
Taxa de fusão & $2500 \mathrm{~kg} \mathrm{/} \mathrm{h}$ \\
Potência do Transformador & $1700 \mathrm{~kW}$ \\
Frequência & $60 \mathrm{~Hz}$ \\
Tensão de entrada & $22000 \mathrm{~V}$ \\
Tensão de saída & $575 \mathrm{~V}$ \\
Potência do Conversor & $1500 \mathrm{~kW}-12 \mathrm{pulsos}$ \\
Regulagem de potência & 0 a 100\% \\
Carga térmica (Sistema de arrefecimento) & $500000 \mathrm{kcal} / \mathrm{h}$ \\
Vazão de água (Sistema de arrefecimento) & $50 \mathrm{~m}$ / h \\
Sistema hidráulico de basculamento & 2 \\
Sistema de exaustão de gases & 2 \\
\hline
\end{tabular}

Fonte: Adaptado do manual de instruções do equipamento.

Fornos elétricos a indução são equipamentos tecnológicos complexos, compostos por inúmeros componentes divididos em subsistemas. Nesse tipo de estrutura os modos de falha competem entre si pela falha geral, portanto o primeiro 
componente ou subsistema que falhar causa a falha geral do sistema. Essas características apontam para o modelo de distribuição de Weibull.

O forno é responsável pela fusão de ligas de aços especiais, suprimento básico para a linha de produção de aços da empresa. O equipamento é único na linha, não havendo substitutos ou redundantes. Dada sua importância, decidiu-se estudar sua confiabilidade. Para tanto, foram escolhidos modelos de distribuição de probabilidade com aderência aos dados de tempo entre falhas e tempo até o reparo. Foi utilizado o software ProConf 2000.

O Proconf 2000 é um aplicativo que faz uso de métodos analíticos e gráficos para ajustar amostras de tempos de falha e de reparo a distribuições de probabilidade. O software testa o ajuste dos dados aos seguintes modelos paramétricos: exponencial negativa, gamma, normal, lognormal e Weibull. Os parâmetros da distribuição são calculados por máxima verossimilhança pelos testes de Qui-Quadrado $\left(\boldsymbol{X}^{2}\right)$ e Kolmogorov-Smirnov (KS) (FRITSCH e RIBEIRO, 1998), amplamente difundidos na literatura. Para cada teste, o software informa o nível de significância e aponta que distribuições não podem ser rejeitadas. A validação é dada se o nível de significância for maior que 5\% em ambos os testes. Cabe ao pesquisador, caso mais do que uma distribuição não possa ser rejeitada, justificar a escolha por uma delas por fundamentação teórica.

Informações complementares sobre o ProConf 2000 e suas rotinas são encontradas em Fritsch e Ribeiro (1998). Mais considerações sobre o teste de máxima verossimilhança podem ser encontradas em Lawless (2003), e melhoramentos do seu uso no modelo de Weibull em Ho e Silva (2005).

Os dados de tempo até o reparo do forno foram testados para as distribuições: exponencial, gamma, normal, lognormal e Weibull pelo software ProConf 2000. Conforme a Tabela 5 , a hipótese de que os dados se ajustem às distribuições lognormal e Weibull não podem ser rejeitadas. Pela premissa da pesquisa, adotou-se o modelo lognormal. 
Tabela 5 - Nível de significância do ajuste aos modelos de distribuição

\begin{tabular}{cccc}
\hline Distribuição & Teste $\boldsymbol{X}^{\mathbf{2}}$ & Teste KS & Decisão \\
\hline Exponencial & $84,97 \%$ & $1,02 \%$ & Rejeitada \\
Gamma & $34,83 \%$ & $4,86 \%$ & Rejeitada \\
Normal & $0,37 \%$ & $0,01 \%$ & Rejeitada \\
Lognormal & $57,64 \%$ & $24,51 \%$ & Não rejeitada \\
Weibull & $43,97 \%$ & $8,41 \%$ & Não rejeitada \\
\hline
\end{tabular}

Fonte: Adaptado do software ProConf 2000

Pela análise gráfica oferecida no ProConf 2000, apresentada na Figura 2, 0 papel de probabilidade do modelo lognormal parece descrever razoavelmente a amostra de dados, ratificando os resultados analíticos.

Figura 2 - Papel de probabilidade lognormal para o TTR

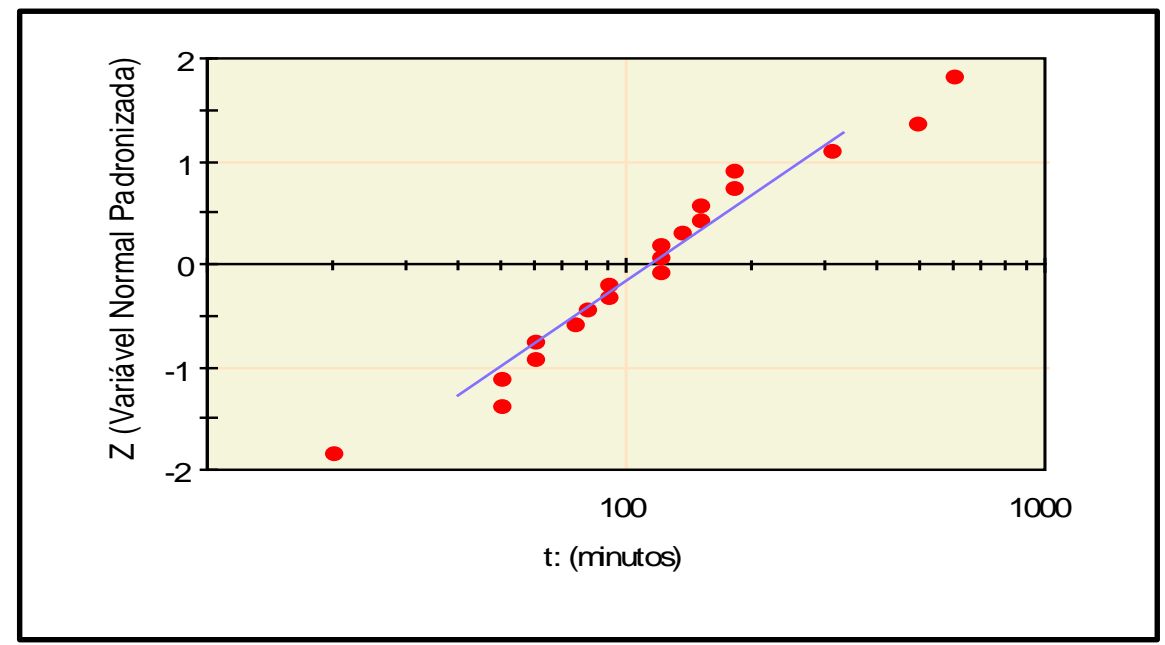

Fonte: ProConf (2000)

Os resultados dos ajustes apresentados pelo ProConf 2000 aparecem na Tabela 6. O tempo médio até o reparo ou Mean Time to Repair (MTTR) do forno é de 156 minutos. Os valores de $t_{10}$ e $t_{50}$ são os limites de tempos em que $10 \%$ e $50 \%$ dos reparos ocorreram. Para um intervalo de confiança de 95\%, o tempo médio até o reparo compreende valores entre 105,37 e 253,23 minutos. A função mantenabilidade $M(t)$ é apresentada na Figura 3. 
Tabela 6 - Modelo lognormal para tempo até o reparo

\begin{tabular}{lc}
\hline Parâmetro & Resultado \\
\hline $\boldsymbol{t}_{10}$ & 23,74 \\
$\boldsymbol{t}_{\mathbf{5 0}}$ & 120,42 \\
MTTR & 156,79 \\
IC 95\% & 105,37 até 253,23 \\
\hline
\end{tabular}

Fonte: Adaptado do software ProConf (2000)

Figura 3 - Função mantenabilidade $M(t)$

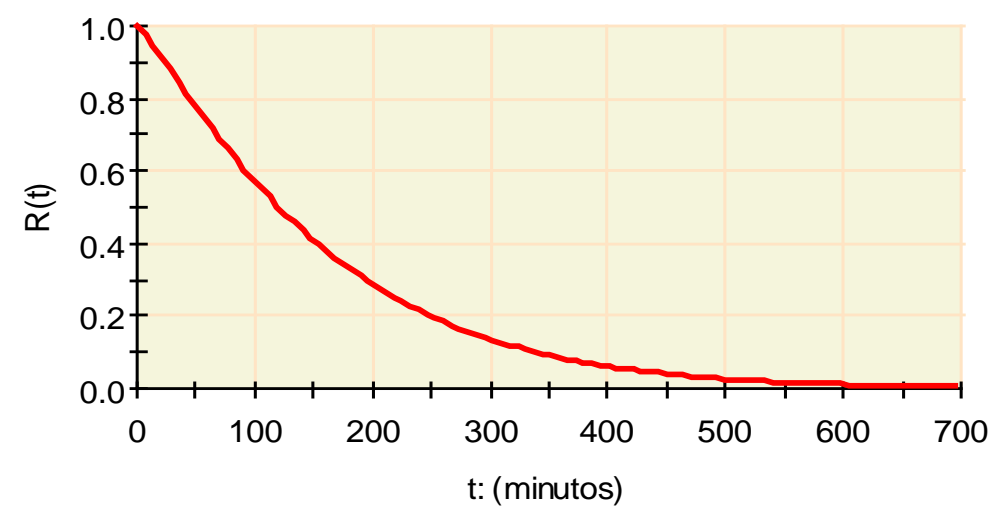

Fonte: ProConf (2000)

De maneira semelhante aos TTR, foram testadas as distribuições exponencial, gamma, normal, lognormal e Weibull para os TBF. Weibull, gamma e exponencial não foram rejeitadas. Pela premissa da pesquisa, adotou-se o modelo exponencial, que corresponde a um caso particular do modelo Weibull, para fator de forma unitário. Os resultados do ProConf 2000 são apresentados na Tabela 7.

Tabela 7 - Nível de significância do ajuste aos modelos de distribuição

\begin{tabular}{cccc}
\hline Distribuição & Teste $\boldsymbol{X}^{2}$ & Teste KS & Decisão \\
\hline Exponencial & $16,66 \%$ & $22,00 \%$ & Não rejeitada \\
Gamma & $8,10 \%$ & $18,28 \%$ & Não rejeitada \\
Normal & $0,05 \%$ & $0,01 \%$ & Rejeitada \\
Lognormal & $3,86 \%$ & $22,52 \%$ & Rejeitada \\
Weibull & $7,95 \%$ & $22,18 \%$ & Não rejeitada \\
\hline
\end{tabular}

Fonte: Adaptado do software ProConf (2000) 
A Figura 4 apresenta a análise gráfica do papel de probabilidade do modelo exponencial para a amostra de dados. Os mesmos parecem se ajustar razoavelmente à distribuição, corroborando com os resultados analíticos.

Figura 4 - Papel de probabilidade exponencial para TBF

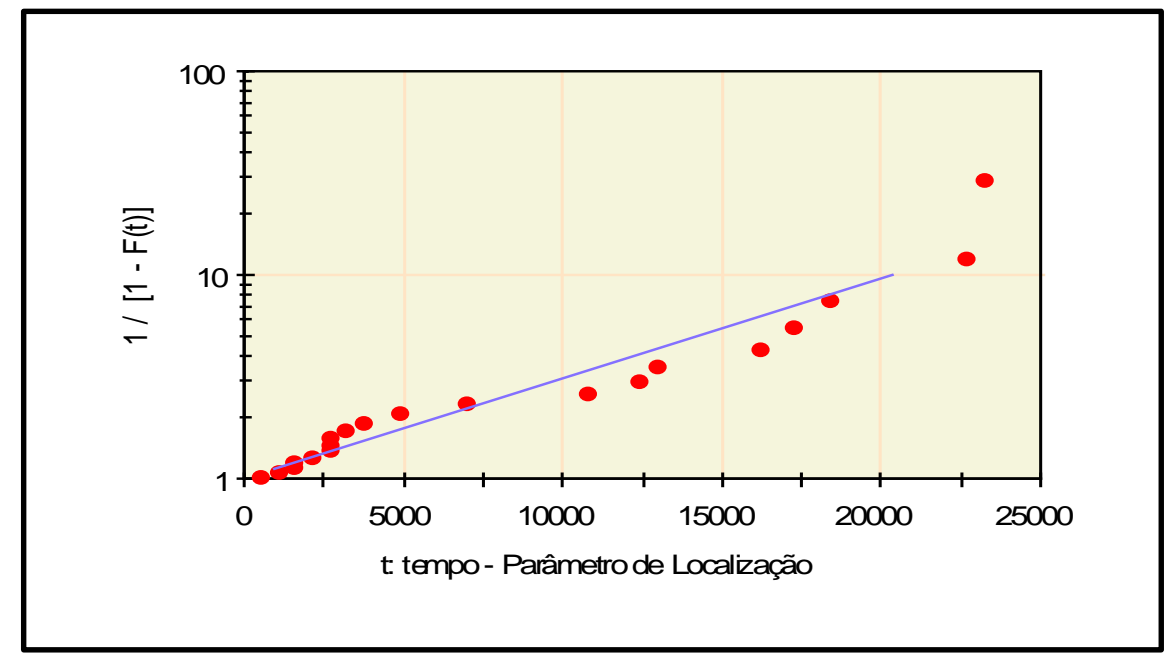

Fonte: ProConf (2000)

Os resultados dos ajustes do ProConf 2000 são apresentados na Tabela 8. Os valores de $t_{10}$ e $t_{50}$ são os limites de intervalos em que $10 \%$ e $50 \%$ das falhas ocorreram. O tempo médio entre falhas ou Mean Time Between Failures (MTBF) do forno é de 8.397 minutos. A função confiabilidade $R(t)$ é apresentada na Figura 5.

Tabela 8 - Modelo exponencial para TBF

\begin{tabular}{lc}
\hline Parâmetro & Resultados \\
\hline $\boldsymbol{t}_{\mathbf{1 0}}$ & 937.8044 \\
$\boldsymbol{t}_{\mathbf{5 0}}$ & 5838.567 \\
Parâmetro de & 59.34 \\
localização & 8.397 \\
MTBF & 0,00012 \\
$\boldsymbol{\lambda}$ & 0,00007 até 0,00018 \\
IC 95\% $(\lambda)$ & \\
\hline
\end{tabular}

Fonte: Adaptado do software ProConf (2000) 
Figura 5 - Função confiabilidade $R(t)$

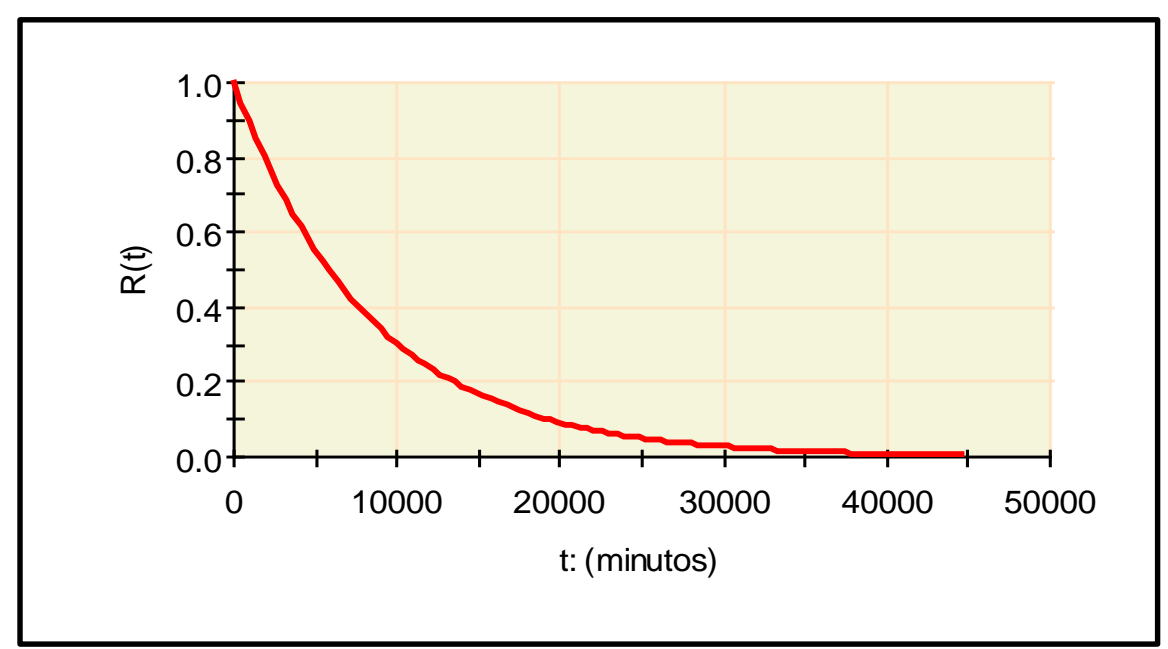

Fonte: ProConf (2000)

Aplicando os valores de MTTR e MTBF, obtidos pelo software ProConf 2000, na equação (24), obtém-se pela equação (25) a disponibilidade do forno.

$$
A v(t)_{A}=\frac{8397}{8397+156}=98,18 \%
$$

Portanto, pode-se afirmar que em $98,18 \%$ do tempo compreendido no estudo o forno esteve disponível para operação.

\subsection{Discussão}

O objetivo desta sessão é discutir como os resultados da pesquisa podem ser usados no gerenciamento da manutenção do equipamento estudado.

A curva da banheira representa genericamente a função de risco $h(t)$ ao longo do ciclo de vida do forno de indução. Obteve-se fator de forma $\gamma=1$, que remete ao modelo exponencial. Com isto, assume-se que o forno em estudo está na maturidade. A taxa de falha é representada pela Figura 6 e a provável posição do equipamento na curva da banheira é apresentada na Figura 7. 
Figura 7 - Taxa de falhas

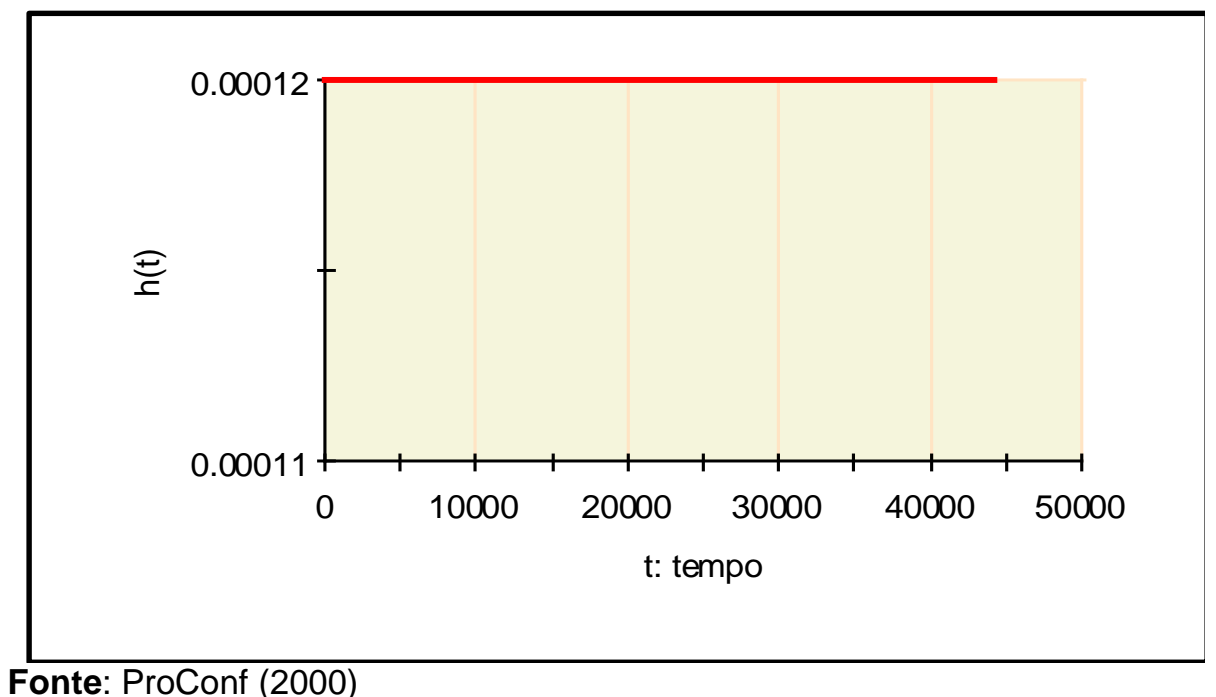

Figura 7 - Posição do forno na curva da banheira

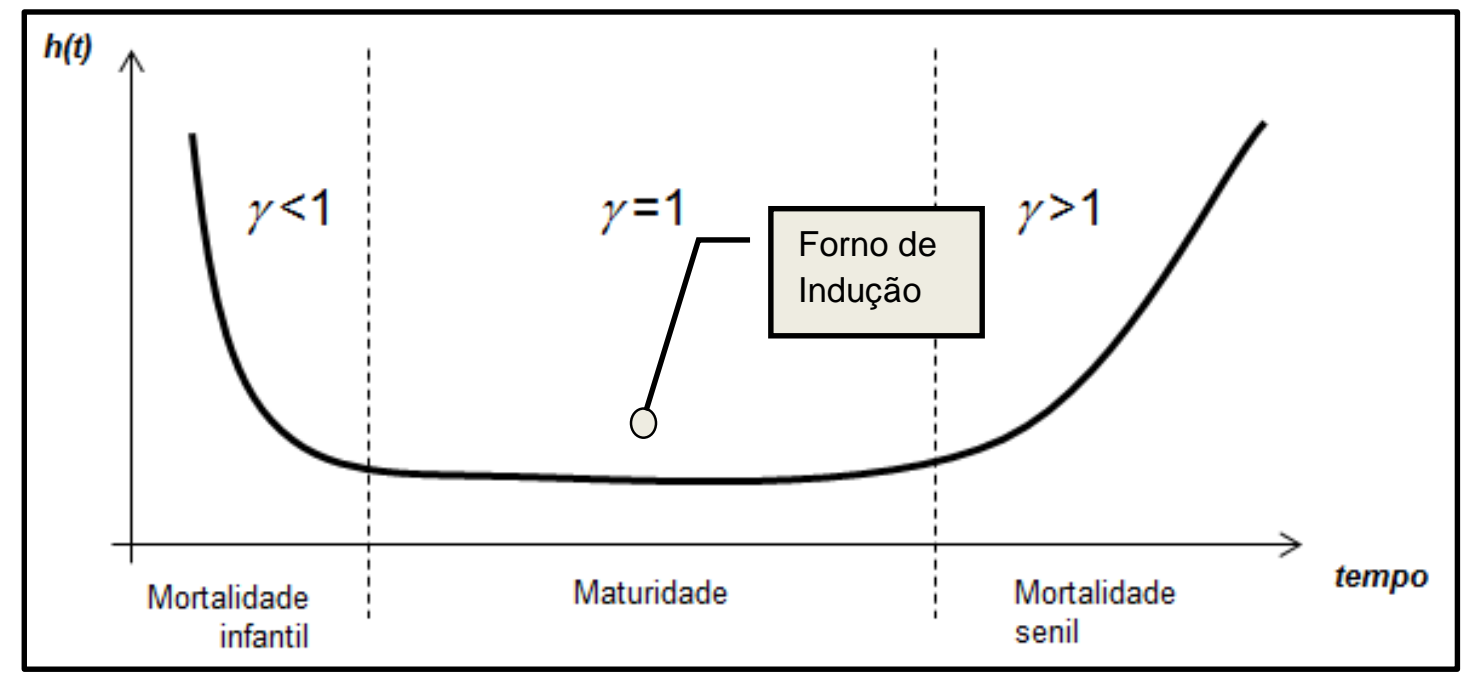

Fonte: Adaptado de Sellitto (2005)

Segundo Sellitto (2005), a melhor estratégia de manutenção para essa fase do ciclo de vida do forno é a manutenção preditiva. Esse tipo de estratégia tem como objetivo monitorar sinais que possam apontar o surgimento de falhas e eventualmente, corrigir e sanar estas falhas antes que se transformem em quebras e em eventuais perdas de produção (MONCHY, 1989; KARDEC e NASSIF, 2001).

A Engenharia de Manutenção da empresa adota a estratégia de manutenção preventiva. Essa estratégia compreende a troca antecipada de componentes que, se ainda não falharam, falharão em curto prazo. Devido à importância e criticidade desse equipamento para o processo produtivo da empresa, os intervalos entre trocas de componentes são baseados nas recomendações dos fabricantes. A empresa adota 
essa estratégia pela premissa de que os custos de paradas não programadas são menores que os da substituição preventiva de componentes.

Porém, outros fatores importantes devem ser observados. A manutenção preventiva substitui prematuramente alguns componentes, aumentando custos com sobressalentes e diminuindo a disponibilidade do equipamento devido à retirada do equipamento de operação para execução de manutenções programadas. Outro ponto negativo é a diminuição da confiabilidade do equipamento. Quando se faz manutenção sem que exista defeito, introduzem-se possibilidades de surgimento de defeitos não existentes no equipamento, tais como: falha humana, falha de sobressalentes, ou falhas nas atividades de manutenção. Em suma, retira-se uma peça que ainda não falhou sem que haja indicativo de falha (taxa de falha constante) e coloca-se outra sobre a qual nada se conhece, pois não há histórico.

Sellitto (2005) faz uma relação entre as diversas estratégias de manutenção e a fase de maturidade, na qual se encontra o forno em estudo. Segundo o autor, ao adotar a manutenção preventiva em um equipamento que esteja na fase de maturidade corre-se o risco de recuo, uma vez que serão trocados os itens sobreviventes, que não apresentaram falhas de origem. Há probabilidade de ganho, pois pode-se introduzir uma peça mais robusta, mas também há uma probabilidade de perda, pois pode-se introduzir uma peça mais frágil e criar um problema onde não existe. No longo prazo, a tendência é cair a confiabilidade e até se recuar na curva, voltando à mortalidade infantil.

Segundo Sellitto (2005), os modos de falhas mais comuns na fase de maturidade têm característica aleatória, causados por falhas de operação, usos incompatíveis, instalação modificada ou por falhas na execução de procedimentos. Com base nos dados de falhas do sistema de informação da empresa, as maiores incidências apuradas foram de falhas relacionadas a entupimentos no sistema de arrefecimento do forno em estudo. Esse tipo de falha causa sobreaquecimento do sistema e o forno se desarma automaticamente para evitar danos maiores. Esses entupimentos ocorrem devido a impurezas que impedem a passagem do líquido refrigerante nas tubulações do sistema de arrefecimento. Conforme constatado pelos técnicos de manutenção da empresa, grande parte das impurezas são fragmentos de ferrugem originados da própria tubulação, o que indica possíveis falhas na execução dos procedimentos de limpeza e filtragem de sólidos. 
Existem técnicas apropriadas para o monitoramento e diagnose de tubulações e fluidos. Dentre elas, pode se indicar a termografia e ferrografia para casos como o deste artigo. A termografia é uma técnica de inspeção preditiva que acompanha as alterações de temperatura ao longo da tubulação em estudo com relação a um padrão preestabelecido. Estas alterações permitem identificar a ocorrência de desgaste ou corrosão na parte interna da tubulação (MIRSHAWKA, 1991; ALVARENGA et al., 2010). Já a ferrografia é uma técnica de manutenção preditiva que analisa e quantifica as partículas de desgaste presentes no fluido em estudo, auxiliando na identificação do tipo desgaste, do contaminante e do desempenho do fluido (NEPOMUCENO, 1989; GRANJA, 1999). Ambas podem fornecer informações capazes de auxiliar em uma possível tomada de decisão quanto ao tipo de intervenção e sua periodicidade.

Informações complementares sobre termografia e ferrografia, além de outras técnicas de manutenção preditiva, podem ser encontradas em Mirshawka (1991) e Nepomuceno (1989).

Observa-se que o foco atual da manutenção é a preservação do equipamento como item físico, não da função produtiva que este exerce no sistema. Como o equipamento é crítico para o sistema produtivo da empresa, podendo ser considerado um gargalo, a Engenharia de Manutenção pode adotar a metodologia da Manutenção Centrada na Confiabilidade (MCC). Segundo a MCC, a manutenção, para desempenhar sua função estratégica, deve estar voltada para os resultados da empresa. Não basta, apenas, reparar o equipamento o mais rápido possível, mas, sim, manter a sua função disponível para operação pelo maior tempo possível e reduzir a probabilidade de paradas não planejadas.

A utilização da MCC pode resultar na redução das atividades de manutenção preventiva e seus respectivos custos. A MCC é um processo contínuo e sua aplicação deve ser periodicamente reavaliada. No início da aplicação, as diretrizes de manutenção devem ser determinadas de modo apriorístico, pois ainda não há informação específica disponível. Porém, à medida que os dados são obtidos, as diretrizes são reavaliadas em bases mais realistas.

\section{CONSIDERAÇÕES FINAIS}

O objetivo deste artigo foi definir uma estratégia de manutenção para um forno elétrico a indução, instalado em uma fundição de aços especiais. As limitações da 
pesquisa foram: estudo de um único equipamento em um único intervalo de tempo de sua vida produtiva, o segundo semestre de 2012. Como o equipamento foi instalado em 2009, atingiu a fase de maturidade e ainda não atingiu a fase de mortalidade senil. Se o estudo for repetido em momento futuro, é possível que os resultados sejam outros.

Foram revisados os conceitos de confiabilidade e mantenabilidade. Em confiabilidade, foram revisadas as funções densidade, densidade acumulada de falhas, confiabilidade e risco. Em mantenabilidade, foram revisadas a função distribuição de reparo e o tempo médio até o reparo com seu reflexo na disponibilidade do equipamento. Também foram revisadas distribuições de probabilidade de maior interesse para a manutenção: normal, lognormal, exponencial, gamma e Weibull.

Quanto a estratégias de manutenção, a revisão focou na curva da banheira (bathtub curve), que representa o comportamento da taxa de falha do equipamento ao longo do seu ciclo de vida. Foram revisados os três períodos característicos e as estratégias mais adequadas para cada um. Também foram revisadas a Manutenção Centrada na Confiabilidade (MCC) e como a manutenção pode ser voltada para o negócio da empresa.

$\mathrm{Na}$ parte prática, o método proposto foi aplicado ao equipamento mais importante do processo produtivo de uma fundição de aços especiais, o forno de fusão por indução elétrica. Foram obtidos, do sistema de informação da empresa, dados de tempos entre falhas e tempos até o reparo do equipamento. Os dados foram analisados com o auxílio do software ProConf 2000. Assumiu-se que os dados coletados fossem confiáveis.

Comparou-se a estratégia de manutenção utilizada pela empresa com a proposta pelo estudo. O estudo indicou que o equipamento está na fase de maturidade, o que remete à estratégia de manutenção preditiva. A estratégia preventiva, utilizada pela empresa, não condiz com a posição do equipamento no ciclo de vida.

Ressalte-se a importância deste estudo para empresa, pois, além desse forno, pertencente à linha de produção de aços especiais, a empresa possui outro semelhante na linha de produção de ligas de ferro fundido. Os fornos são equipamentos críticos para o processo produtivo da empresa: sua parada representa a parada da sua respectiva linha de produção, tal como usualmente ocorre em indústrias de processamento contínuo (SELLITTO, 2002). 
Observa-se também que a disponibilidade dos equipamentos pode ser um importante indicador para estratégias de manutenção, pois equipamentos críticos, com capacidade restritiva, com maior disponibilidade permitem lotes maiores de produção a custos unitários menores. Esses ganhos em escala são fundamentais para empresas de fundição que competem por preço. Por esse motivo, a MCC é indicada a este caso, já que seu princípio é manter a função do sistema disponível pelo maior tempo possível.

Conclui-se que análises quantitativas dos tempos entre falhas, tempos até o reparo e da disponibilidade do equipamento podem ser importantes na definição da estratégia de manutenção da empresa, pois possibilitam aos gestores visualizarem o reflexo das ações de manutenção na disponibilidade do sistema.

Por fim, para possível continuidade da pesquisa, sugere-se a análise de confiabilidade e disponibilidade da linha de produção como um todo, com a construção de um diagrama de blocos de confiabilidade (Reliability Block Diagram - RBD), utilizando, por exemplo, a análise de confiabilidade em sistemas apresentada em Lafraia (2001) e Fogliatto e Ribeiro (2009), auxiliada pelo software ProSis 2000, e para a disponibilidade do sistema, o Método das Cadeias de Markov, apresentado em Lewis (1996), Lafraia (2001) e Dodson e Nolan (2002).

\section{AGRADECIMENTOS}

Parte da pesquisa foi financiada pelo CNPq. Agradece-se aos revisores anônimos pelas sugestões.

\section{REFERÊNCIAS}

ASSOCIAÇÃO BRASILEIRA DE NORMAS TÉCNICAS (ABNT) - NBR 5462: Confiabilidade e mantenabilidade. Rio de Janeiro, 1994.

BERNARDES, M. Caracterização do modelo probabilístico dos tempos entre falhas em linhas de transmissão. Trabalho de Conclusão de Curso (Engenharia de Produção), UNISINOS, São Leopoldo: 2006.

CHTECH. ProConf 2000: Confiabilidade de componentes. Porto Alegre: $\mathrm{CHTech}$ Desenvolvimento de sistemas LTDA, 2000.

CHTECH. ProSis 2000: Confiabilidade de Sistemas em Série ou Paralelo. Porto Alegre: CHTech Desenvolvimento de sistemas LTDA, 2000.

CORRÊA, H.; CORRÊA, C. Administração de produção e operações: manufatura e serviços: uma abordagem estratégica. São Paulo: Atlas, 2010. 
CUNHA, M.; VILLAS-BOAS, F.; KAMINSKI, P. Considerações sobre Confiabilidade no Projeto de Submarinos. Produto \& Produção, v.13 n.1, p.114-130, 2012.

DANTAS, M.; VALENÇA, D.; FREIRE, M.; MEDEIROS, P.; SILVA, D.; ALOISE, D. Modelo de regressão Weibull para estudar dados de falha de equipamentos de sub-superfície em poços petrolíferos. Produção, v.20, n.1, p.127-134, 2010. http://dx.doi.org/10.1590/S010365132010005000010

DODSON, B. Weibull Analysis. Milwaukee: ASQ, 1994.

DODSON, B.; NOLAN, D. Reliability engineering handbook. Tucson: QA Publishing, 2002.

FAGUNDES, A.; ROCHA, A.; BARBOSA, S.; CARVALHO, A. Estudo de caso: análise quantitativa de confiabilidade e disponibilidade de um torno $\mathrm{CNC}$, baseado na metodologia RCM (Reliability Centred Maintenance), aplicado a área de manutenção industrial. Anais do XXXI ENEGEP, Encontro Nacional de Engenharia de Produção. Belo Horizonte: ABEPRO, 2011.

FILIPPINI, R. Operations management research: some reflections on evolution, models an empirical studies in OM. International Journal of Operations and Production Management, v.26, n.7, p.655-670, 1997. http://dx.doi.org/10.1108/01443579710175583

FOGLIATTO, F.; RIBEIRO, J. Confiabilidade e manutenção industrial. Rio de Janeiro: Elsevier, 2009.

FRITSCH, C.; RIBEIRO, J. PROCONF: Um software orientado para análises de confiabilidade. In. XVIII Encontro Nacional de Engenharia de Produção. Niterói, 1998.

GIRELLI, A.; HOFFELDER, M.; SEVERO, M.; SILVA, V. VACCARO, G. Otimização de sobressalentes e disponibilidade operacional em plantas petroquímicas. Anais do XXVI ENEGEP, Encontro Nacional de Engenharia de Produção. ABEPRO, Fortaleza: 2006.

GROSH, D. A primer of reliability theory. New York: John Wiley \& Sons, 1989.

HO, L.; SILVA, A. Estimadores não viciados para o tempo médio até a falha e para percentis obtidos do modelo de regressão Weibull. Gestão \& Produção, v.12, n.1, p.97-105, 2005. http://dx.doi.org/10.1590/S0104-530X2005000100009

KARDEC, A.; NASCIF, J. Manutenção: função estratégica. Rio de Janeiro: Qualitymark, 2001.

KUYVEN, P. Métodos estatísticos aplicados ao processo decisório. São Leopoldo: Editora Unisinos, 2010.

LAFRAIA, J. Manual de confiabilidade, mantenabilidade e disponibilidade. Rio de Janeiro: Qualitymark, 2001.

LAWLESS, J. Statistical models and methods for lifetime data. New York: John Wiley \& Sons, 2003.

LEWIS, E. Introduction to reliability engineering. New York: John Wiley \& Sons, 1996.

MARTINS, R. Abordagens quantitativa e qualitativa. In: MIGUEL, P. (org.), Metodologia de pesquisa em Engenharia de Produção e Gestão de Operações. Rio de Janeiro: Campus, 
2010.

MENDES, A. Manutenção Centrada em Confiabilidade: uma abordagem quantitativa. Dissertação (Mestrado em Engenharia de Produção), Universidade Federal do Rio Grande do Sul - UFRGS, Porto Alegre, RS, 2011.

MENGUE, D.; SELLITTO, M. Estratégia de manutenção baseada em funções de confiabilidade para uma bomba centrífuga petrolífera. Produção Online, v.13, n.2, p.759783, 2013. http://dx.doi.org/10.14488/1676-1901.v13i2.1341

MIGUEL, P. Adoção do estudo de caso na Engenharia de Produção. In: MIGUEL, P. (org.), Metodologia de pesquisa em Engenharia de Produção e Gestão de Operações. Rio de Janeiro: Campus, 2010. http://dx.doi.org/10.1590/S0103-65132007000100015

MIGUEL, P. Estudo de caso na engenharia de produção: estruturação e recomendações para sua condução. Produção, v.17, n.1, p.216-229, 2007. http://dx.doi.org/10.1590/S010365132007000100015

MONCHY, F. A função manutenção: formação para a gerência da manutenção industrial. São Paulo: Durban/EBRAS, 1989.

MONTGOMERY, D.; RUNGER, G.; HUBELE, N. Estatística aplicada à engenharia. Rio de Janeiro: LTC, 2011.

MORABITO, R.; PUREZA, V. Modelagem e simulação. In: MIGUEL, P. (org.), Metodologia de pesquisa em Engenharia de Produção e Gestão de Operações. Rio de Janeiro: Campus, 2010.

NAKANO, D. Métodos de pesquisa adotados na Engenharia de Produção e Gestão de Operações. In: MIGUEL, P. (org.), Metodologia de pesquisa em Engenharia de Produção e Gestão de Operações. Rio de Janeiro: Campus, 2010.

NELSON, W. Applied life data analisys. New York: John Wiley \& Sons, 1982. http://dx.doi.org/10.1002/0471725234

PACHECO, D.; ANTUNES JÚNIOR, J.; LACERDA, D.; GOLDMEYER, D.; GILSA, C.; Modelo de gerenciamento da capacidade produtiva: integrando teoria das restrições e o índice de rendimento operacional global (IROG). Produção Online, v.12, n.3, p. 806-826, 2013. http://dx.doi.org/10.14488/1676-1901.v12i3.981

RAMOS FILHO, J.; ATAMANCZUK, M.; MARÇAL, R. Seleção de técnicas de manutenção para processo de armazenagem pelo Método de Análise Hierárquica. Produção Online, v.10, n.1, p.142-166, 2010. http://dx.doi.org/10.14488/1676-1901.v10i1.375

RAPOSO, C. Overall Equipment Effectiveness: aplicação em uma empresa do setor de bebidas do polo industrial de Manaus. Produção Online, v.11, n.3, p.648-667, 2010. http://dx.doi.org/10.14488/1676-1901.v11i3.529

REIS, L.; ANDRADE, J. Análise de falhas e da posição na curva da banheira de moldes empregados em equipamentos de injeção. In. XXIX Encontro Nacional de Engenharia de Produção. Salvador, 2009.

SANTOS, W.; COLOSIMO, E.; MOTTA, S. Tempo ótimo entre manutenções preventivas para sistemas sujeitos a mais de um tipo de evento aleatório. Gestão \& Produção, v.14, n.1, p.193-202, 2007. http://dx.doi.org/10.1590/S0104-530X2007000100016 
SELLITTO, M. Análise estratégica da manutenção de uma linha de produção metal-

mecânica baseada em cálculos de confiabilidade de equipamentos. GEPROS, v.3, n.2, p.97108, 2007.

SELLITTO, M. Formulação estratégica da manutenção industrial com base na confiabilidade dos equipamentos. Produção, v.15, n.1, p.044-059, 2005. http://dx.doi.org/10.1590/S010365132005000100005

SELLITTO, M. Inteligência artificial: uma aplicação em uma indústria de processo contínuo. Gestão \& Produção, v.9, n.3, p.363-376, 2002. http://dx.doi.org/10.1590/S0104530X2002000300010

SELLITTO, M.; BORCHARDT, M.; ARAÚJO, D. Manutenção centrada em confiabilidade: aplicando uma abordagem quantitativa. Anais do XXII ENEGEP, Encontro Nacional de Engenharia de Produção. Curitiba: ABEPRO, 2002.

SILVA, A.; ANZANELLO, M. Análise de confiabilidade para a redução do índice de refugo em um processo de calandragem. Trabalho de Conclusão de Curso (Engenharia de Produção), UFRGS, Porto Alegre: 2012.

SIQUEIRA, I. Manutenção centrada na confiabilidade: manual de implementação. Rio de Janeiro: Qualitymark, 2009.

SLACK, N, HARRISON, A.; HARLAND, C.; JOHNSTON, R.; CHAMBERS, S.

Administração da produção. São Paulo: Atlas, 2010.

WUTTKE, R.; SELLITTO, M. Cálculo da disponibilidade e da posição na curva da banheira de uma válvula de processo petroquímico. Produção Online, v.8, n.4, p.1-23, 2008. http://dx.doi.org/10.14488/1676-1901.v8i4.134

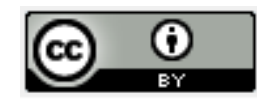

Artigo recebido em 09/06/2013 e aceito para publicação em 28/03/2014

DOI: http://dx.doi.org/ 10.14488/1676-1901.v15i2.1615 